\title{
Theorethical efficiency of a gear based azimuthal tracked photovoltaic platform
}

\author{
B. Butuc ${ }^{1}$, G. Moldovean ${ }^{1}$ and N. Creanga ${ }^{1}$ \\ ${ }^{1}$ Department of Renewable Energy Systems and Recycling \\ Transilvania University of Brasov \\ Eroilor 29, 500036, Brasov, Romania, \\ Phone/Fax number:+40 268 412088, fax +40 268410525 e-mail: bianca.butuc@unitbv.ro, ghmoldovean@unitbv.ro, \\ nora.creanga@unitbv.ro
}

\begin{abstract}
The paper presents the results of a theoretical analysis developed to estimate the energy produced by an azimuthal tracked photovoltaic (PV) system. The mechanical structure of the tracking system is build up by a worm gear serial connected to a double straight bevel gear. Sun tracking is attained through the sun's trajectories tracking method.

There are presented, simulated and comparatively analysed 5 series of tracking programs, characterized by different numbers of annual intervals and of the step performing intervals. The analysis is made in the hypothesis of clear sky for Braşov Romania geographical coordinates: $45.65^{\circ} \mathrm{N}$ latitude, $25.58^{\circ} \mathrm{E}$ longitude.
\end{abstract}

The conclusions can be directly used in the implementation stage of the optimum tracking program, to experimentally verify the energetic response theoretically estimated.

\section{Key words}

Azimuthal tracking system, tracking discreet program, tracking efficiency

\section{Introduction}

The energy received by the earth's surface can substitute the amount of the energy obtained from fossil fuels [1]. Therefore, the photovoltaic conversion systems are widely used in various applications.

A main factor influencing the efficiency of the PV conversion systems is the solar radiation rate of use. So, azimuthal tracking systems are a viable technical solution often used to maximize the amount of solar radiation falling on the PV surface $[2,3]$.

A proper design of the tracked PV conversion systems requires evaluating the energetic performances considering the infield conditions of the implementation site.
The main objective of this paper is to estimate the energetic efficiency of an azimuthal tracked PV system build up of a worm gear -T1 serial connected with a double straight bevel gear $-\mathrm{T} 2$. The proposed tracking system, described in [4] and presented in Figure 1, is the subject of a patent request registered at OSIM Bucureşti, Romania with the number A/740/21.09.09.

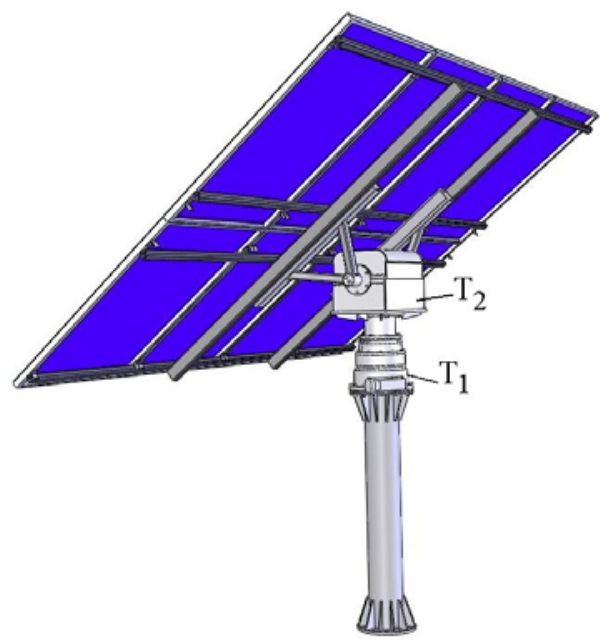

Fig. 1. Gear based azimuthal tracked PV system

A PV tracking system is considered to be energy efficient when the following relation [5] is satisfied:

$$
\varepsilon=\left(E_{T}-E_{F}\right)-E_{c}>>0
$$

where $E_{T}$ is the energy produced by the tracked PV surface, $E_{F}$ is the energy produced by the same surface without being tracked and $\mathrm{E}_{\mathrm{C}}$ is the energy consumed to perform the tracking movements. $\mathrm{E}_{\mathrm{T}}$ is determined considered as input data the tracking method, respectively the tracking programs. 
Sun tracking is attained through the sun's trajectories tracking method. The main advantages of this method are related to the simplicity of the control scheme, the substantial energy gain and the possibility of tracking programs optimization [6].

A tracking program influences the energetic efficiency of a PV system. So, it is important that the models used to estimate the energy performances of an azimuthal PV system to reach high accuracy in prediction while the number of the input parameters is minimal.

In this paper are presented, simulated and analysed 5 tracking programs (I, II, III, IV, V), characterized by different numbers of annual intervals and performing steps intervals, as presented in Table I.

The analysis is performed in the hypothesis of clear sky for Braşov - Romania geographical coordinates: $45.65^{\circ} \mathrm{N}$ latitude, $25.58^{\circ} \mathrm{E}$ longitude.

Table I. - Tracking programs

\begin{tabular}{|c|c|c|c|c|c|c|c|c|c|c|}
\hline $\begin{array}{c}\text { Tracking } \\
\text { programs }\end{array}$ & \multicolumn{2}{|c|}{ I } & \multicolumn{2}{|c|}{ II } & \multicolumn{2}{c|}{ III } & \multicolumn{2}{c|}{ IV } & \multicolumn{2}{|c|}{ V } \\
\hline $\begin{array}{c}\text { Annual } \\
\text { intervals }\end{array}$ & \multicolumn{2}{|c|}{4} & \multicolumn{2}{|c|}{6} & \multicolumn{2}{|c|}{8} & \multicolumn{2}{|c|}{10} & \multicolumn{2}{|c|}{12} \\
\hline $\begin{array}{c}\text { Steps time } \\
\text { length [h] }\end{array}$ & $1 / 2$ & 1 & $1 / 2$ & 1 & $1 / 2$ & 1 & $1 / 2$ & 1 & $1 / 2$ & 1 \\
\hline
\end{tabular}

\section{Tracking programs modelling}

The principle of the tracking method is based on the input data related to sun's position described by the altitudinal $-\alpha$ and azimuthal $-\psi$ [7] angles through the following relations:

$$
\begin{gathered}
\sin \alpha=\sin \delta \sin \varphi+\cos \delta \cos \varphi \cos \omega \\
\cos \psi=\frac{\sin \alpha \sin \varphi-\sin \delta}{\cos \alpha \cos \varphi}
\end{gathered}
$$

where $\varphi$ represents the site latitude, $\delta-$ the declination angle and $\omega$ hour angle.

A discreet (in steps) tracking implies a change in the position of the PV surface. The altitudinal $\alpha^{*}$ and azimuthal $\psi^{*}$ specific rotational angles are modified at equal time intervals (Figure 2). The angles describing the PV surface position are established according to their sun homologues angles $\alpha$ and $\psi$.

Compared to screw-nut based tracking systems, the angular strokes of the proposed system $\Delta \alpha^{*}$ and $\Delta \psi^{*}$, have the same variation domain as the daily sun's angular strokes $\Delta \alpha$ and $\Delta \psi$.

Due to its mechanical characteristics the system angular stroke $\Delta \psi^{*}$ performed around the vertical axes is in the range of $\pm 180^{\circ}$, while the limits of the $\Delta \alpha^{*}$ angular stroke are $0^{\circ} \ldots 90^{\circ}$.
By combining the two movements described by the $\alpha^{*}$ and $\psi^{*}$, the daily sun movement from $\mathrm{E}$ to $\mathrm{W}$ and the seasonal movement from $\mathrm{S}$ to $\mathrm{N}$ are followed. So, the plane of the photovoltaic surface is continuously set in a normal position against the solar ray vector.

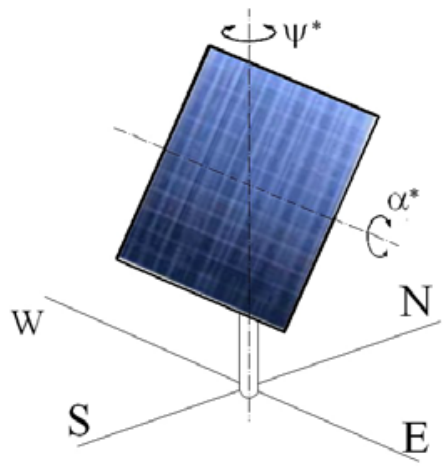

Fig. 2. The specific rotational angles of the azimuthal system

The $\Delta \alpha^{*}$ and $\Delta \psi^{*}$ specific rotational angles are modified according to the tracking programs. The tracking programs are developed based on the simplified method presented in [6]. So, the year was divided into 4, 6, 8, 10 and 12 annual interval, set symmetrically about the curve of solar altitudinal angle variation. For each annual interval were considered the middle day and the lower and upper limits days. For example, in Figures 3 and 4 are presented the limit days of the annual intervals established for tracking programs I and II.

The proposed tracking programs have different step time lengths and are based on different annual intervals. The variation of $\alpha^{*}$ and $\psi^{*}$, along with the steps time length is constant over an annual interval.

The limit days of the tracking programs are:

1) Program I: the limit days are presented in Figure 2a;

2) Program II: $\mathrm{N} \in\{[31-80]$, [81-125], [126-218], [219-261], [262-309], [310-31]\};

3) Program III: the limits of the annual intervals are presented in Figure 2b;

4) Program IV: $\mathrm{N} \in\{[32-59]$, [60-89], [90-116], [117-146], [147-198], [199-228], [229-255], [256-284], [285-313], [314-31]\};

5) Program $V: \mathrm{N} \in\{[24-46],[47-67],[68-90],[91-$ 114], [115-143], [144-199], [200-227], [228252], [253-275], [276-297], [298-318], [319$23]\}$.

In Figure 5 is presented the variation of the proposed system angles in the middle and limit days of the fourth annual interval of the IV tracking program. Even though the variation of the solar angles $\alpha$ and $\psi$ is different in the limit days of the annual interval, the variation of $\alpha^{*}$ and $\psi^{*}$, set optimum in the middle day, is the same for all the days of the annual interval. 


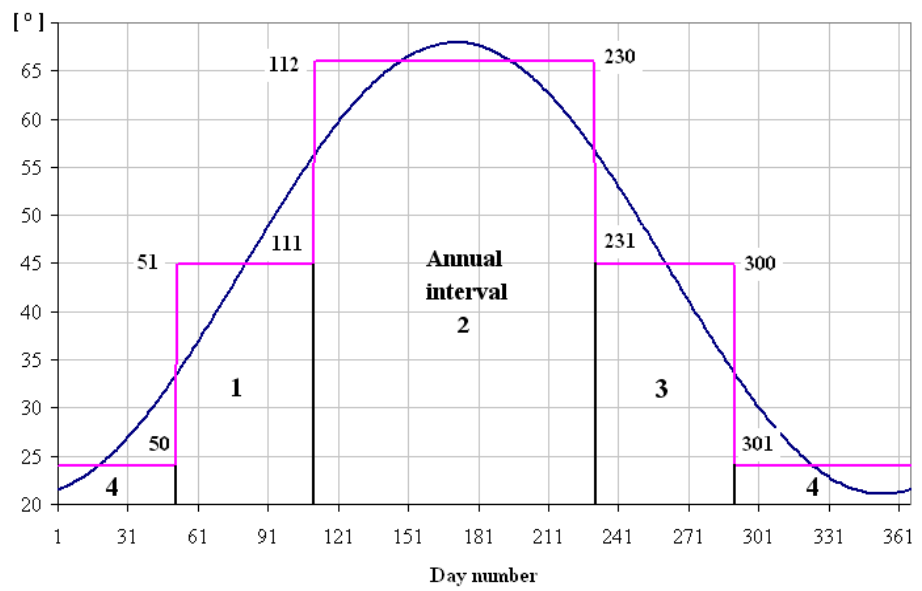

Fig. 3. The annual intervals for the tracking program I

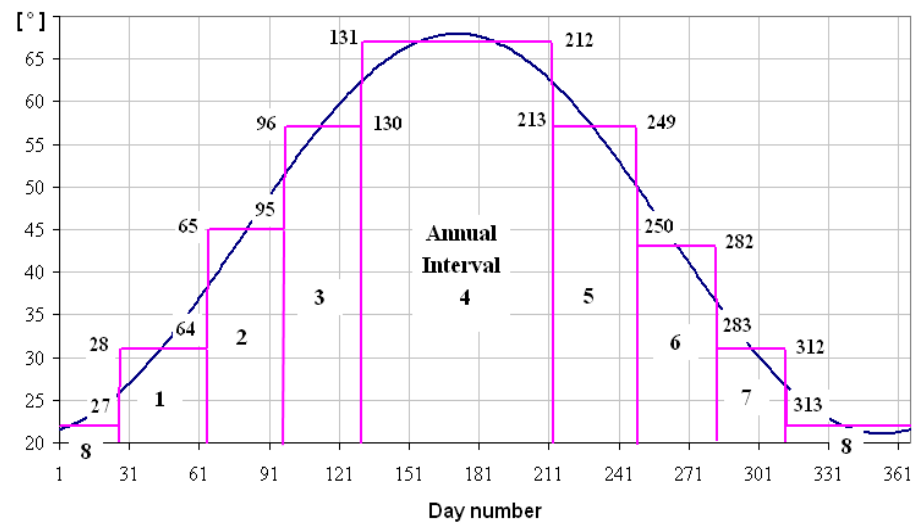

Fig. 4. The annual intervals for the tracking program III

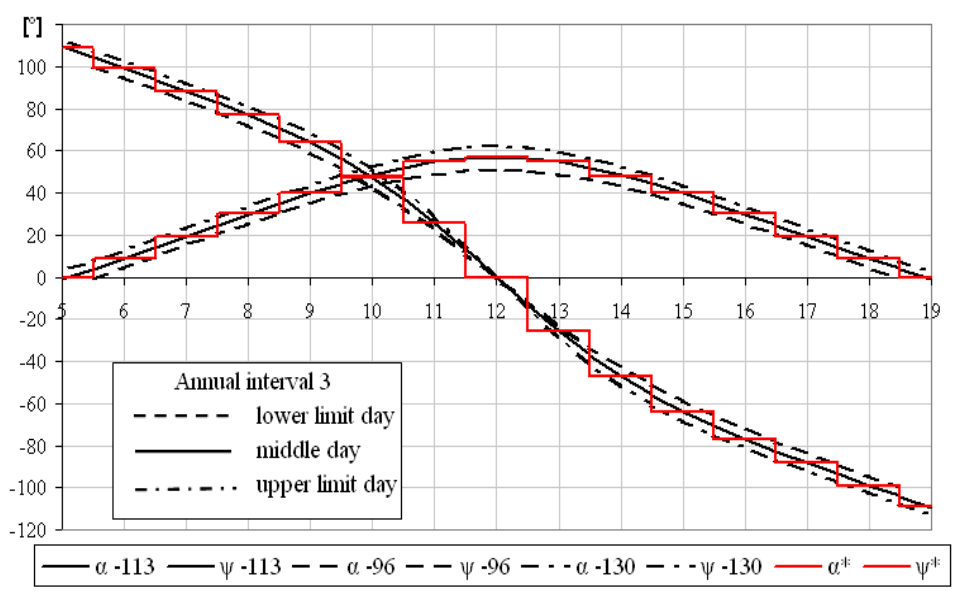

Fig. 5. The variation of the angles specific to the azimuthal system for the III

\section{Numerical simulation of tracking programs efficiencies}

The estimation of system energy response, considering the 5 tracking programs, involves the determination of the quantity of direct solar radiation, respectively of the energy produced by the system.

In terms of photovoltaic conversion process the useful direct solar radiation component $\mathrm{B}_{P V}$ is reduced by the cosine of the angle described by the solar ray unit vector and the normal unit vector of the surface, called the incidence angle $v[7]$ :

$$
B_{P V}=B_{M} \cos v
$$

where $\mathrm{B}_{M}$ represents the available direct solar radiation, modelled according to [9]:

$$
B_{M}=G_{0} \exp \left(-\frac{T_{R}}{0.9+9.4 \sin \alpha}\right)
$$




$$
G_{0}=1367\left(1+0.034 \cos \left(0.9856^{\circ} N-2.72\right)\right)
$$

where $\mathrm{G}_{0}$ is the extraterrestrial radiation, $\mathrm{T}_{\mathrm{R}}-$ the turbidity factor and $\mathrm{N}$ is the day number of the year. The variation of turbidity factor changes in time and space, depending on climatic and geographic conditions of the site. A typical value for this turbidity factor for Europe is 3 [10].

In the case of an azimuthal system, the incidence angle is determined based on the information related to sun-earth geometry and PV surface position. The analytical model developed in $[10,11]$, gives the incidence angle relation as follows:

$$
\begin{aligned}
& \cos v=\bar{e}_{\text {sun-ray }} \cdot \bar{e}_{P V-a z}= \\
& =\cos \alpha \cdot \cos \alpha^{*} \cdot \cos \left(\psi-\psi^{*}\right)+\sin \alpha \cdot \sin \alpha^{*}
\end{aligned}
$$

where $e_{\text {sun-ray }}$ is the solar unit ray and $e_{P V-a z}$ is the PV surface normal unit vector.

In the local system of sun ray projection, the incidence angle is influenced by the parameters describing the sun apparent movement on the sky $\alpha$ and $\psi$, and the ones describing the specific angles of the azimuthal PV system $\alpha^{*}$ and $\psi^{*}$. These parameters are the input data in the command and control system.

The incidence angle variation was simulated for each tracking program, both for steps performed at half hour time interval and one hour time intervals. In Figure 6 is presented its variation for the fifth annual interval of the III tracking program. The limit days of this interval are 262 and 309.
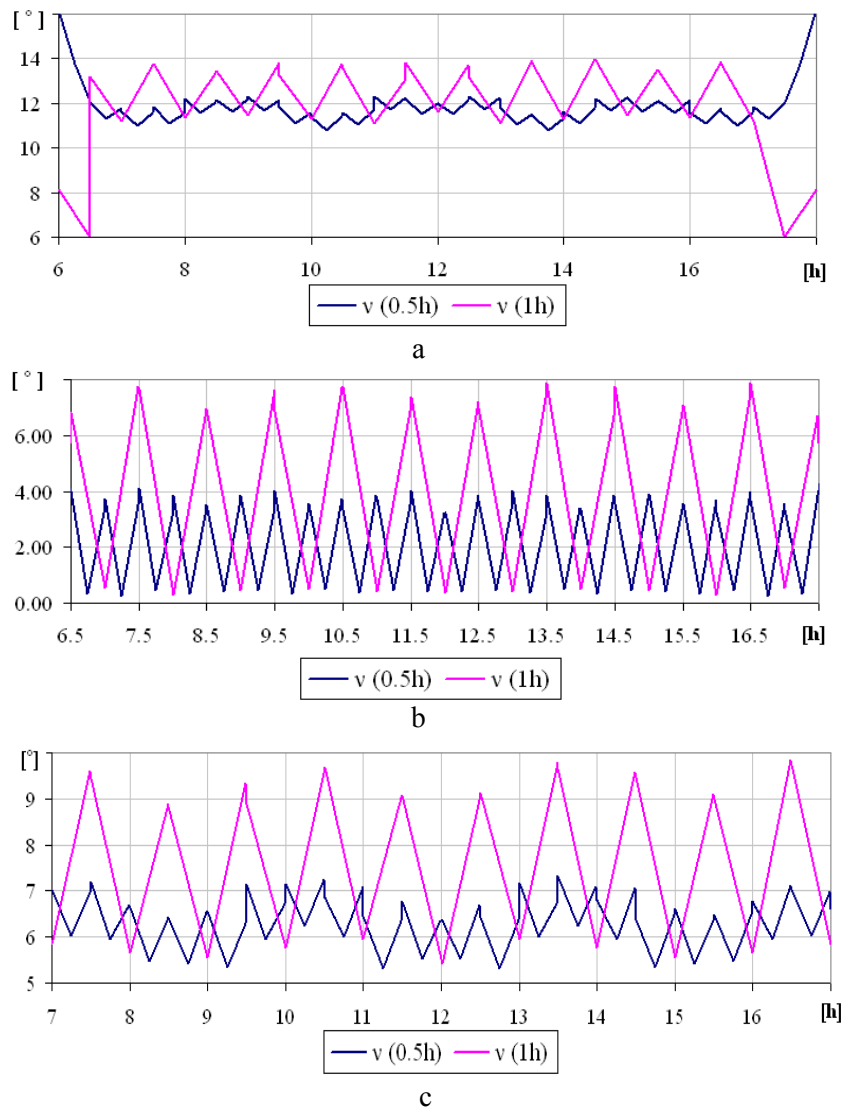

Fig. 6. Incidence angle variation for the fifth annual interval of the tracking program III: a -lower limit day, b - middle day, c - upper limit day

It can be noticed that the variation range is low, between $0^{\circ}-8^{\circ}$ in the middle day, for steps performed at one hour time interval. For steps performed at every half an hour time interval the limit values of the incidence angle are higher. In the limit days of the annual interval the incidence angle ranges between $5^{\circ}-16^{\circ}$.

Due to the cosine reduction of the useful direct solar radiation component, the influence of the incidence angle variation on the quantity of the direct solar radiation was analysed.
For example, in Figure 7 it is presented the solar radiation received by the $\mathrm{PV}$ surface in the middle and limit days of the fifth annual interval of the III tracking program. The lower values of the incidence angle in the middle day have a minor influence on the quantity of the direct solar radiation.

The variation of the instantaneous values of the direct solar radiation is similar to the variation of the instantaneous values of the available direct solar radiation. In the limit days, due to the incidence angle 
high deviation, the values of the instantaneous received direct solar radiation are lower than the instantaneous values of the available direct solar radiation.

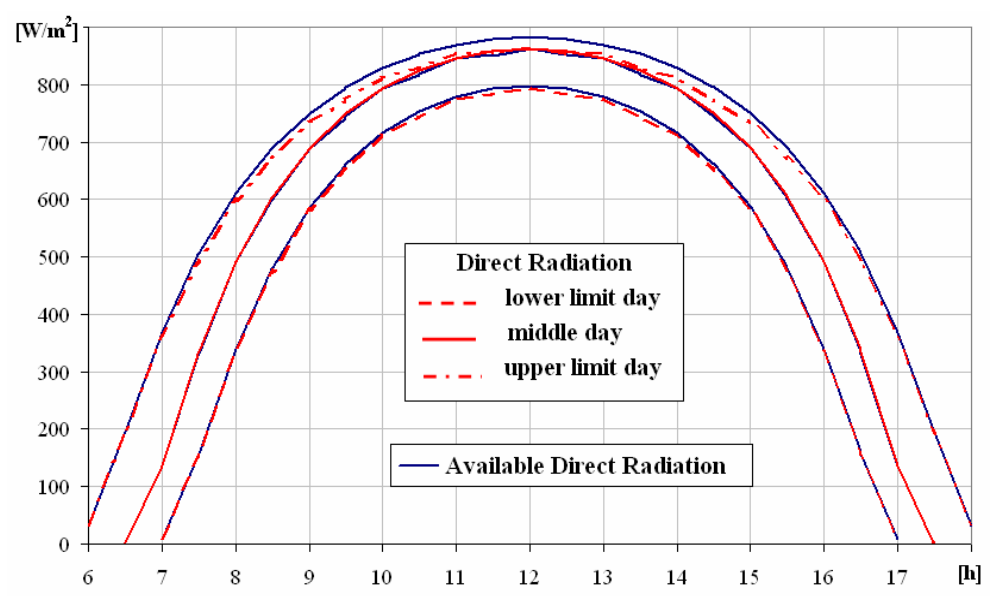

Fig. 7. Available direct solar radiation $B_{M}$ and direct solar radiation on the $P V$ surface $B_{P V}$ in the key days of the fifth annual interval of the III tracking program, for one hour steps performing interval

The variations of the instantaneous available solar direct radiation and received direct solar radiation, and the energy response were simulated for each tracking program. The results were used in the analyses of the annual interval numbers influence on the tracking efficiency. Tracking efficiency was considered to be the ratio between the quantity of the direct solar radiation and the available direct solar radiation. The tracking efficiency values obtained for each annual interval of each tracking program are presented in Table II.

Table II. - Tracking programs efficiencies

\begin{tabular}{|c|c|c|c|}
\hline & \multirow{2}{*}{$\begin{array}{l}\text { Annual } \\
\text { interval }\end{array}$} & \multicolumn{2}{|c|}{ Tracking efficiency [\%] } \\
\hline & & $1 \mathrm{~h}$ & $0.5 \mathrm{~h}$ \\
\hline \multirow{4}{*}{ 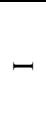 } & 1 & 98.4 & 97.8 \\
\hline & 2 & 98.6 & 98 \\
\hline & 3 & 98 & 97.4 \\
\hline & 4 & 98.6 & 98.1 \\
\hline \multirow{6}{*}{$\Xi$} & 1 & 98.7 & 98.4 \\
\hline & 2 & 99.2 & 98.6 \\
\hline & 3 & 99.3 & 98.8 \\
\hline & 4 & 99.2 & 98.6 \\
\hline & 5 & 98.9 & 98.3 \\
\hline & 6 & 99.4 & 98.9 \\
\hline \multirow{8}{*}{$\Xi$} & 1 & $\overline{99.4}$ & 98.9 \\
\hline & 2 & 99.3 & 98.8 \\
\hline & 3 & 99.5 & 98.9 \\
\hline & 4 & 99.5 & 99 \\
\hline & 5 & 99.5 & 98.8 \\
\hline & 6 & 99.4 & 98.8 \\
\hline & 7 & 99.6 & 99 \\
\hline & 8 & 99.5 & 99 \\
\hline \multirow{10}{*}{$\geq$} & 1 & 99.6 & 99.1 \\
\hline & 2 & 99.3 & 98.8 \\
\hline & 3 & 99.5 & 98.9 \\
\hline & 4 & 99.7 & 99.1 \\
\hline & 5 & 99.7 & 99.2 \\
\hline & 6 & 99.7 & 99.1 \\
\hline & 7 & 99.5 & 98.9 \\
\hline & 8 & 99.5 & 98.9 \\
\hline & 9 & 99.6 & 98.9 \\
\hline & 10 & 99.5 & 99 \\
\hline
\end{tabular}

Table II. - Tracking programs efficiencies

\begin{tabular}{|c|c|c|c|}
\hline \multirow{2}{*}{$\begin{array}{c}\text { Annual } \\
\text { interval }\end{array}$} & \multicolumn{2}{|c|}{ Tracking efficiency [\%] } \\
\cline { 2 - 3 } & $1 \mathrm{~h}$ & $0.5 \mathrm{~h}$ \\
\hline 1 & 99.7 & 99.1 \\
2 & 99.7 & 99 \\
3 & 99.6 & 98.9 \\
4 & 99.3 & 99 \\
5 & 99.6 & 99.1 \\
6 & 99.8 & 99.2 \\
7 & 99.7 & 99.2 \\
8 & 99.6 & 98.9 \\
9 & 99.6 & 98.9 \\
10 & 99.6 & 98.9 \\
11 & 99.7 & 99 \\
12 & 99.6 & 99.1 \\
\hline
\end{tabular}

\section{Discussions and conclusions}

The paper presents the results of the analyses performed to determine the theoretical energy efficiency of an azimuthal PV system build up of a worm gear serial connected with a double bevel gear. Using the proposed tracking system high angular strokes can be achieved, ensuring this way high energy efficiency.

The tracking efficiencies were comparatively analysed considering that the PV surface position is modified at every half an hour and an hour time intervals. The results, presented in a graphical and tabular manner, highlight the influence of the number of annual intervals on the azimuthal PV system tracking efficiency (Figure 8).

An increase in annual intervals number results in an increased tracking efficiency, maximum value being obtained for the $\mathrm{V}$ tracking program. A high tracking efficiency can be also attained by performing the steps at every half an hour time interval. 
Although a higher tracking efficiency is reached for steps performed at half an hour time intervals, deciding on the optimum tracking program involves detailed studies regarding the consumed energy.

A proper correlation between the quantity of the direct solar radiation and the energy consumed to perform the steps $\mathrm{E}_{\mathrm{C}}$ ensures the maximization of the photovoltaic conversion. Therefore, the energy consumed per each step represents the main factor influencing the decision on choosing the optimum tracking program.

The conclusions can be used in the implementation process of the optimum discreet tracking program, in order to experimentally validate the predicted energy efficiency.

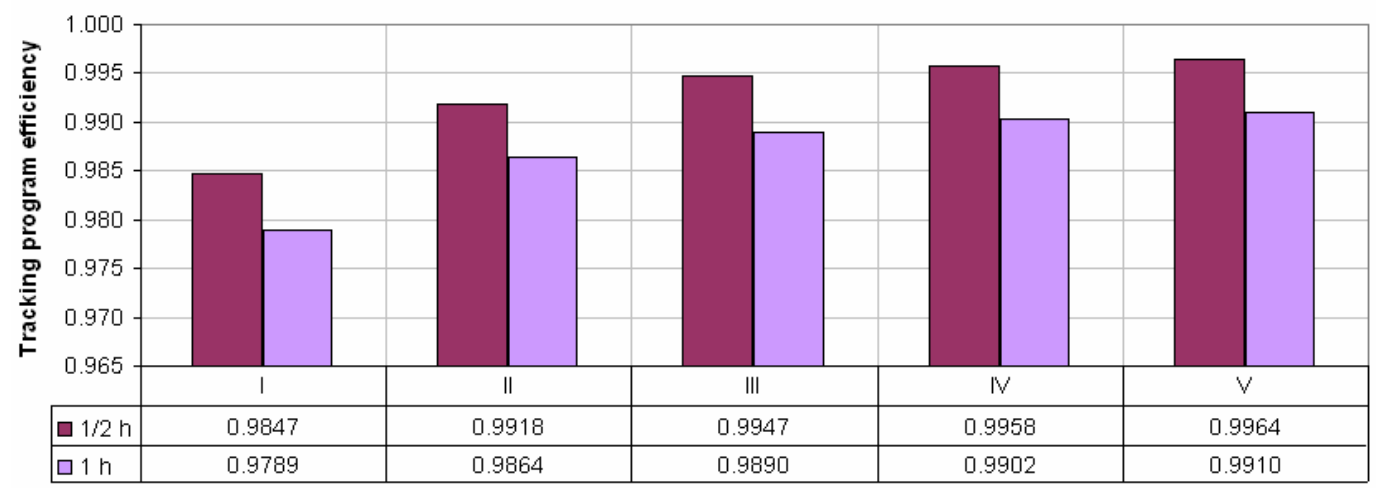

Fig. 8. Tracking efficiencies of the tracking programs developed

Authors further research will focus on the determination of the energy consumed in order to perform the movements and of the energy produced by a non-tracked PV system, so the theoretical efficiency of the system to be estimated.

\section{Acknowledgement}

This paper is supported by the Sectoral Operational Programme Human Resources Development (SOP HRD), financed from the European Social Fund and by the Romanian Government under the contract number POSDRU/6/1.5/S/6".

\section{References}

[1] A. H. Weston, "Quantifying global exergy resources", in Energy, 2006, Vol. 31, pp. 1685-1702.

[2] U. Rindelhardt, A. Dietrich and C. Rösner, "Tracked megawatt PV plants: Operation results 2008 in Germany and Spain", in Proc. of 24th European Photovoltaic Solar Energy Conference, 2009

[3] H. Mousazadeh, "A review of principle and sun-tracking methods for maximizing solar systems output." in Renewable and Sustainable Energy Reviews 2009, Vol. 13, pp.1800-1818.
[4] G. Moldovean, B. Butuc, R. Velicu, "Tracking system with a single motor", in New Trends in Mechanism Science, 2010, pp. 649-656.

[5] C. Alexandru, "Modeling and Simulation of the Tracking Mechanism Used for a Photovoltaic Platform", in New Trends in Mechanism Science, 2010, pp. 575-582.

[6] V. Popa, I. Vişa, and D. Diaconescu, "Tracking program optimisation for a PV azimuthal system", in 24th European Photovoltaic Solar Energy Conference 2009, pp. 3680-3683.

[7] R. Messenger, and J. Ventre, Photovoltaic System Engineering, London (2000), pp.21-39.

[8] M. Vătăşescu, I. Vişa, D. Diaconescu, I. Hermenean, and N. Creangă, "New solar angles and their corresponding tracking systems efficiency", in Proc. ICREPQ 2010.

[9] M. Meliss, Regenerative Energiequellen-Praktikum, Springer Verlag Berlin 1997.

[10] F. Bason,'’Diffuse Solar Irradiance and Atmospheric Turbidity", in Proc EuroSun 2004.

[11] I. Vişa, et al, "The incidence Angles of the trackers used for the PV panels' Orientation. Part II: Azimuthal Trackers", in Proc.ICEEMS 2007-International Conference on Economic Engineering and Manufacturing Systems, Vol.8, pp. 375-380.

[12] I. Vişa, et al, "On the incidence angle optimization of the dual-axis solar trackers", in Proc of 11th International Research/Expert Conference "Trends in the Development and Machinery and Associated Technology" TMT 2007, pp. 11111114. 